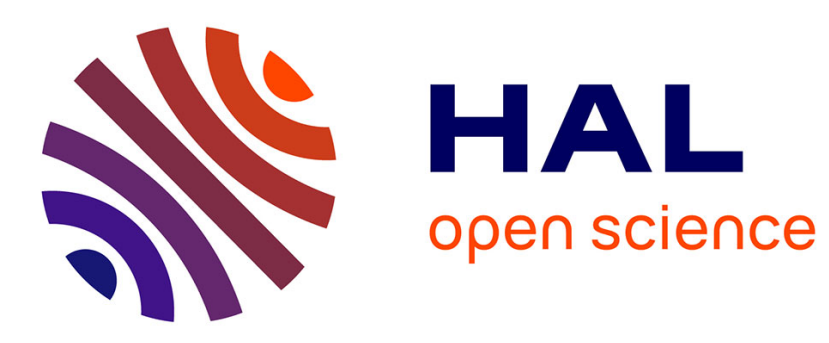

\title{
Inference and decision in credal occupancy grids: use case on trajectory planning
}

\author{
Marie-Hélène Masson, Sébastien Destercke, Véronique Cherfaoui
}

\section{To cite this version:}

Marie-Hélène Masson, Sébastien Destercke, Véronique Cherfaoui. Inference and decision in credal occupancy grids: use case on trajectory planning. International Journal of Uncertainty, Fuzziness and Knowledge-Based Systems, 2021, 29 (4), pp.537-557. 10.1142/S0218488521500239 . hal-03341136

\section{HAL Id: hal-03341136 https://hal.science/hal-03341136}

Submitted on 10 Sep 2021

HAL is a multi-disciplinary open access archive for the deposit and dissemination of scientific research documents, whether they are published or not. The documents may come from teaching and research institutions in France or abroad, or from public or private research centers.
L'archive ouverte pluridisciplinaire HAL, est destinée au dépôt et à la diffusion de documents scientifiques de niveau recherche, publiés ou non, émanant des établissements d'enseignement et de recherche français ou étrangers, des laboratoires publics ou privés. 
December

4

2020

Inference and decision in credal occupancy grids: use case on trajectory planning

Marie-Hélène Masson. UMR CNRS 7253 Heudiasyc Université de Picardie Jules Verne, France mmasson@hds.utc.fr

Sébastien Destercke

UMR CNRS 7253 Heudiasyc

Sorbonne Université, Université de Technologie de Compiègne CS 60319 - 60203 Compiègne cedex, France sebastien.destercke@hds.utc.fr

Véronique Cherfaoui

UMR CNRS 7253 Heudiasyc

Sorbonne Université, Université de Technologie de Compiègne CS 60319 - 60203 Compiègne cedex, France veronique.cherfaoui@hds.utc.fr

Received (received date)

Revised (revised date)

\begin{abstract}
Occupancy grids are common tools used in robotics to represent the robot environment, and that may be used to plan trajectories, select additional measurements to acquire, etc. However, deriving information about those occupancy grids from sensor measurements often induce a lot of uncertainty, especially for grid elements that correspond to occluded or far away area from the robot. This means that occupancy information may be quite uncertain and imprecise at some places, while being very accurate at others. Modelling finely this occupancy information is essential to decide the optimal action the robot should take, but a refined modelling of uncertainty often implies a higher computational cost, a prohibitive feature for real-time applications. In this paper, we introduce the notion of credal occupancy grids, using the very general theory of imprecise probabilities to model occupancy uncertainty. We also show how one can perform efficient, real-time inferences with such a model, and show a use-case applying the model to an autonomous vehicle trajectory planning problem.
\end{abstract}

Keywords: Occupancy grids and Trajectory planning and Imprecision and Imprecise probabilities

\title{
1. Introduction
}

37 Occupancy grids are commonly used tools to represent a mobile robot (cars, drones, 
vehicle, they are instrumental in solving issues such as obstacle avoidance and shortterm trajectory planning, and are particularly suitable in dynamic environments, for instance environments discovered on the fly by the robots (such as in SLAM problems 27) or environments in which moving obstacles evolves 19. Such grids are composed of multiple cells, which in the most usual settings can be either free or occupied. Those grids are most of the time obtained from sensor readings associated to processing steps that may be peculiar to each sensor, according to their nature (cameras, lasers, ....). Such occupancy information is therefore prone to be affected by many sources of uncertainties, such as imprecise sensor readings (camera occlusion, numerical precision, ..., ), noise due to environmental conditions (rain, ...) or faulty sensors. Different frameworks have been proposed to model and reason from such uncertainties, for instance by using probabilistic $21 \mid 6$, fuzzy $28|24| 23$ or evidential $20[33 \sqrt[26 / 30]{30}$ occupancy grids.

In this paper, we explore how another uncertainty framework, namely imprecise probabilities, can be used to model uncertainty in occupancy grids. To our knowledge, it is the very first attempt to do so. Imprecise probability consists in extending the classical probabilistic model by considering convex sets of probabilities or equivalent models such as lower expectation. This representation formally includes all the other aforementioned representations 10 (possibilities, probabilities, belief functions), and has the benefit to be fully consistent with classical Bayesian probabilistic modelling, both axiomatically (as it relaxes Bayesian axioms) and formally speaking. It can therefore be interpreted either as an uncertainty theory of its own, or as a robustification of the classical probabilistic models. We refer to 2 for an introduction to various aspects of the framework, and to Walley's book 32 for a full theoretical account. In the case of cells taking values in the binary space $\mathcal{X}=\{x, \neg x\}$ composed of the states occupied ( $x$ in our notation) and free ( $\neg x$ in our notation), imprecise probabilistic models encode uncertainty by explicitly encoding imprecision through the use of two boundary measures, therefore allowing to differentiate between what is commonly called aleatoric uncertainty (due to a possible ambiguity and/or random phenomena) and epistemic uncertainty (due to an absence of information).

The concept of imprecise probabilistic occupancy grids is introduced in Section2, where we also discuss the associated challenges in terms of inference and decision. We then consider in Section 3 the specific problem of making inferences for local trajectory planning, proposing a computationally efficient and simple solution to do so. Each step of the method is illustrated using simple examples. Then, in Section 4 for illustrative purposes, we present some results obtained using real occupancy grids coming from PACPUS 17, an experimental platform developed in our laboratory, dedicated to researches on autonomous vehicles. 


\section{Introducing credal occupancy grids}

In this section, we will introduce the notion of credal occupancy grids, and will discuss the problem of making general inferences with them. Roughly speaking, such inferences come down to make computations over (pseudo-)Boolean functions where the probabilities of literals are imprecisely specified. We will however not treat this very general issue that is encountered in many fields such as data-bases ${ }^{13}$, gametheory ${ }^{25}$, but will focus on the most common issue in applications using occupancy grids, that is guiding a moving object through it.

For this reason, we will consider from now that our problem concerns a moving robot, and that the robot environment is modelled by a large grid of cells, and that each cell $X$ can take a binary value, occupied $(x)$ or unoccupied $(\neg x)$. We assume that when a robot perceives its environment through measurement devices (sensors, cameras), it receives uncertain information about whether a given cell is occupied. Imprecise probabilities ${ }^{32}$ offer a very generic way to model uncertainty, including most known models of uncertainty such as precise probabilities, belief functions or possibility distributions 10. It also comes with a fully-fledged theory to reason with such uncertainty and make decisions 32 .

In a binary setting such as the occupancy of cell $X$, any imprecise probabilistic model can be reduced to an interval $[p(x), \bar{p}(x)]$ of the probability to be occupied, as we have that $p(\neg x)=1-\bar{p}(x)$ and similarly for the upper bound. Note that the fact that any convex probability set can be reduced to such intervals on singletons is only true in the binary case, and is used here as one of the key feature that makes our approach tractable. We can in particular model the following extreme situations:

- certain occupancy: $[p(x), \bar{p}(x)]=[1,1]$,

- certain inoccupancy: $[p(x), \bar{p}(x)]=[0,0]$,

- unknown occupancy: $[\underline{p}(x), \bar{p}(x)]=[0,1]$,

as well as all the intermediate ones, with precise probabilities being retrieved when $p(x)=\bar{p}(x)$. A credal occupancy grid is pictured in Figure 1. where cells are voluntary of very large size for illustrative purposes. Green cells are likely to be unoccupied, red ones occupied, and grey/dark ones are in between (i.e., $0.5 \in[\underline{p}(x), \bar{p}(x)]$ ). The darker the shades, the wider are the corresponding intervals.

Let us now consider a set $X_{1}, \ldots, X_{m}$ of cells. As they are equivalent to Boolean variables, any event $A$ concerning these cells (e.g., "at least one cell is occupied"," $k$ consecutive cells are occupied") can be expressed as a Boolean formula, and in particular can be put in Disjunctive Normal Form (DNF). This means that we can express any event in the form of a disjunction $A=\vee_{i=1}^{j} A_{i}$, where each $A_{i}=$ $\wedge_{k \in A_{i}^{+}} x_{k} \wedge \wedge_{k \in A_{i}^{-}} \neg x_{k}$ is a conjunction of values for the cells ( $A_{i}^{+}$denotes the set of indices of positive variable, $A_{i}^{-}$the indices of negative one). Such sets are sometimes called orthopairs ${ }^{5}$ in the literature. When the uncertainty over cells is expressed by a precise probability, assessing $P(A)$ then amounts to evaluate $P\left(\cup_{i=1}^{j} A_{i}\right)$, a 


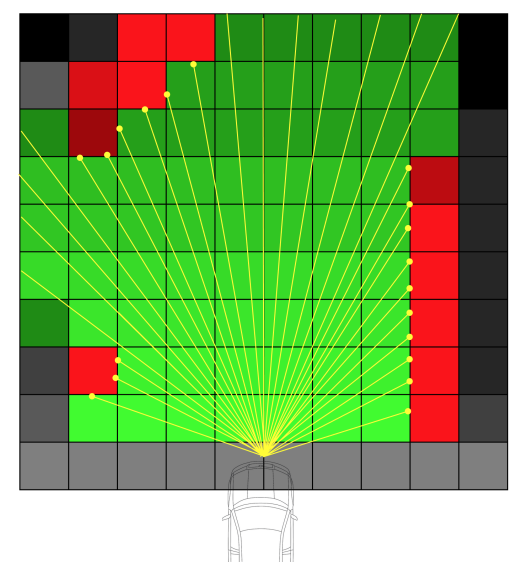

Fig. 1. Credal occupancy grid build from sensor data. Certain occupancy: $[p(x), \bar{p}(x)]=[1,1]$ in red, certain inoccupancy: $[\underline{p}(x), \bar{p}(x)]=[0,0]$ in green, unknown occupancy: $[\underline{p}(x), \bar{p}(x)]=[0,1]$ in black. The intermediate states are represented with lower intensity values.

potentially difficult task. One common way to do it is to use the following formula

$$
P(A)=P\left(\cup_{i=1}^{j} A_{i}\right)=\sum_{\mathcal{A} \subseteq\left\{A_{1}, \ldots, A_{j}\right\}}-1^{|\mathcal{A}|} P\left(\cap_{A \in \mathcal{A}} A_{i}\right)
$$

where the second equality simply applies the inclusion/exclusion principle. The probability of a conjunctive event $A_{i}$ is

$$
P\left(A_{i}\right)=\prod_{k \in A_{i}^{+}} p\left(x_{k}\right) \prod_{k \in A_{i}^{-}} p\left(\neg x_{k}\right) .
$$

and likewise for intersection of such events, that remain of the same form. Solving Equation (1), hence making inferences over a probabilistic grid, is then relatively straightforward when probabilities are precise, as assessing the probabilities of conjunctions is easy. It should however be noted that there are multiple ways to decompose a given event $A$ into a DNF, such as the use of Binary Decision Diagrams ${ }^{4}$ or of minimal cuts in reliability theory ${ }^{16}$, that can lead to more or less computationally friendly decompositions, in the sense that the number of terms in the right-hand side of (2) can grow exponentially. Obtaining compact Boolean representations of an event $A$ is therefore a whole area research, that is outside of the scope of the present paper, which is why we will focus on the second part on specific cases for which efficient decompositions do exist.

However, even when one has obtained a decomposition through some means, the task of solving Equation (2) becomes much more difficult when probabilities

a Note that in this paper, we will use interchangeably $\vee$ and $\cup$, or $\wedge$ and $\cap$, as they have the same meaning. 
become imprecise, as we then have to solve the equation

$$
\underline{P}(A)=\inf _{p\left(x_{i}\right) \in\left\{\underline{p}\left(x_{i}\right), \bar{p}\left(x_{i}\right)\right\}} P(A)
$$

to get the lower bound of the probability of an event $A$, which is NP-hard in general, as Equation 1 is a multi-linear form of the $p\left(x_{i}\right)$, each constrained to be in an interval (see for instance 3 ). Indeed, if it is known 12 that the bound given by Equation 3 is obtained in such a case on one of the vertices of the hyper-cube $\times_{i}\left[p\left(x_{i}\right), \bar{p}\left(x_{i}\right)\right]$, that is $p\left(x_{i}\right) \in\left\{p\left(x_{i}\right), \bar{p}\left(x_{i}\right)\right\}$, it remains to find which one, hence to check for $2^{m}$ combinations if $m$ is the number of cells. The upper bound can be found similarly, replacing inf by sup, with the same difficulties. This means, in particular, that reasoning over an entire imprecise probabilistic grid and for arbitrary events will be computationally prohibitive and infeasible for real-time applications.

Example 1. Let us consider two cells $X_{1}, X_{2}$, and the event "one and only one cell is occupied", that corresponds to a XOR logical gate, that is

$$
A=\left(x_{1} \wedge \neg x_{2}\right) \vee\left(x_{2} \wedge \neg x_{1}\right)=A_{1} \cup A_{2}
$$

with $A_{1} \cap A_{2}=\emptyset$, as each contains opposite values (e.g., $x_{1}$ for $A_{1}$, and $\neg x_{1}$ for $\left.A_{2}\right)$. Let us now consider that $p\left(x_{1}\right) \in[0.6,0.8]$ and $p\left(x_{2}\right) \in[0.3,0.6]$, we have that $\underline{P}(A)$ is obtained on a combination of extreme points, i.e.,

$$
\begin{aligned}
\underline{P}(A) & =\inf _{p\left(x_{i}\right) \in\left\{\underline{p}\left(x_{i}\right), \bar{p}\left(x_{i}\right)\right\}} p\left(x_{1}\right)\left(1-p\left(x_{2}\right)\right)+\left(1-p\left(x_{1}\right)\right) p\left(x_{2}\right) \\
& =0.8 \cdot 0.4+0.2 \cdot 0.6=0.44
\end{aligned}
$$

obtained for $p\left(x_{1}\right)=0.8$ and $p\left(x_{2}\right)=0.6$ (the upper bounds of $p\left(x_{1}\right)$ and $p\left(x_{2}\right)$ ).

More generally, one may be interested not only in computing the lower and upper bounds of some event probabilities, but to compute the lower and upper expected values of some reward/penalty function $f: \times_{k=1}^{m}\left\{x_{k}, \neg x_{k}\right\} \rightarrow \mathbb{R}$. Since it is defined on a discrete space, we can always define a partition $B_{1}, \ldots, B_{k}$ such that $f$ is constant over elements of this partition, i.e. if we denote by $\mathbf{x}$ an assignment vector over cells $X_{i}, f(\mathbf{x})=\mathbf{f}\left(\mathbf{x}^{\prime}\right)$ whenever $\mathbf{x}, \mathbf{x}^{\prime} \in B_{i}$ for some $i \in\{1, \ldots, k\}$. It then makes sense to denote by $f_{B_{i}}$ the value of $f$ for all elements included in $B_{i}$. Computing the lower expectation of $f$ than amounts to solve the equation

$$
\underline{\mathbb{E}}(f)=\inf _{p\left(x_{i}\right) \in\left\{\underline{p}\left(x_{i}\right), \bar{p}\left(x_{i}\right)\right\}} \sum_{j=1}^{k} f_{B_{j}} P\left(B_{j}\right)
$$

with each $B_{j}$ that can be decomposed using (2). As (4) remains a multi-linear form, the problem for solving it is in general not more difficult than the one of finding a lower probability, and its tractability will depend on both the form of $f$ and of the events $B_{i}$.

Example 2. Let us pursue Example 1, by considering a simple game: we get a reward of 2 if the two variables end up with the same value, and suffer a penalty of 


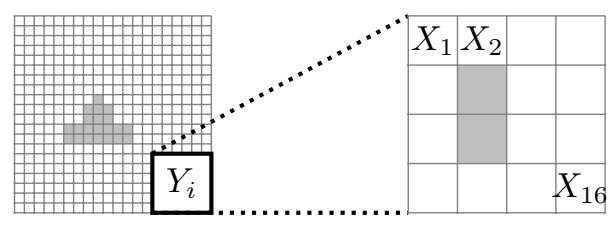

Fig. 2. The metagrid $Y_{i}$ composed of 16 cells of the credal occupancy grid

1 (equivalently, a reward of -1 ) if they have different values. Our knowledge about the variables $X_{1}, X_{2}$ remains the same. One may think, for instance, of a game with two coins with ill-known probabilities of landing head and tail.

We now want to know our lower expected reward, should we play this game. We have $B_{1}=\left(x_{1} \wedge x_{2}\right) \vee\left(\neg x_{1} \wedge \neg x_{2}\right)$ and $B_{2}$ its complement, with $f_{B_{1}}=2$ and $f_{B_{2}}=-1$. We must now minimize

$$
2\left(p\left(x_{1}\right) \cdot p\left(x_{2}\right)+p\left(\neg x_{1}\right) \cdot p\left(\neg x_{2}\right)\right)-\left(p\left(\neg x_{1}\right) \cdot p\left(x_{2}\right)+p\left(x_{1}\right) \cdot p\left(\neg x_{2}\right)\right)
$$

which gives $\underline{\mathbb{E}}(f)=-0.86$, obtained for the values $p\left(x_{1}\right)=0.8$ and $p\left(x_{2}\right)=0.6$.

In the rest of the paper, we will propose a decision model that focuses on particular types of events, that are of practical interest in robot guidance problems and are computationally tractable, in the sense that bound (3) can be computed in a time that is linear in the number of considered cells (or Boolean variables).

\section{Moving in credal occupancy grid: model, inference and decision}

Now that we have introduced credal occupancy grids and discussed their main aspects regarding inference, let us focus on the problem of guiding a moving robot within such an occupancy grid.

From now on, we will also be interested in particular states of subgrids of the whole occupancy grid, that we will call metagrids. Such metagrids will describe the successive locations of a robot along a chosen path. Indeed, usually the space occupied by a robot (be it a vehicle, a drone, etc.) is much bigger than a single cell, whose area is usually related to the smallest zone a robot can perceive with sufficient reliability. As an example, cells of grids for autonomous vehicles are typically squares of a few decimetres A metagrid $Y_{i}$ will be composed of $n$ cells denoted $X_{1}, \ldots, X_{n}$, while a possible path for the robot will always be composed of $k$ metagrids $Y_{1}, \ldots, Y_{k}$, representing its successive positions. The concept of metagrid within a grid is shown in the Figure 2 (with a metagrid being made of 16 elementary cells).

Given this, our decision model will be based on the following elements, that we will detail in different subsections:

- For each metagrid $Y_{i}$, assess the uncertainty over the fact that at least one cell of the metagrid is occupied: this provides lower/upper bounds $\left[\underline{p}\left(y_{i}\right), \bar{p}\left(y_{i}\right)\right]$ over a new Boolean state (either at least one cell is occupied, 
A first inference we want to do is to know, for each metagrid, what is the likelihood of having at least one cell $X_{i}$ that is occupied, as having one such cell is judged sufficient for the metagrid to be unreachable. In practice, this comes down to assess the probability bounds for the event

$$
Y=x_{1} \vee x_{2} \vee \ldots \vee x_{n}
$$

One advantage of this event is that it satisfies a monotonicity constraint, i.e., it cannot become false if one variable $X_{i}$ goes from the value $\neg x_{i}$ to $x_{i}$. It results that the corresponding multi-linear form given by Equation (2) is increasing ${ }^{\text {b }}$ in each variable $p\left(x_{i}\right)$. Previous results about such multi-linear forms 12 , applied for instance in 1 to the case of reliability functions, then indicate that $\underline{P}(Y)$ is obtained by computing $P(Y)$ when $p\left(x_{i}\right)=p\left(x_{i}\right)$. Yet it should be noticed that the form $x_{1} \vee x_{2} \vee \ldots \vee x_{n}$ is not very computationally friendly, as every pair of elements of the disjunction have a non-empty intersection, since no pair of formulas contains opposite values of variables (each formula is only made of positive values). This means that Equation (1) contains a number of terms that factorially increases with $n$. However, we can re-express $Y$ as a disjunction of non-intersecting events (using, e.g., ordered binary decision diagrams 4 )

$$
Y=x_{1} \vee\left(\neg x_{1} \wedge x_{2}\right) \vee\left(\neg x_{1} \wedge \neg x_{2} \wedge x_{3}\right) \vee \ldots \vee\left(\wedge_{i=1}^{n-1} \neg x_{i} \bigwedge x_{n}\right)
$$

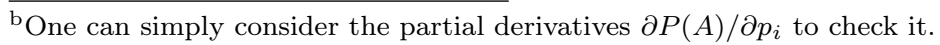




\begin{tabular}{c|cccc}
\hline & $X_{1}$ & $X_{2}$ & $X_{3}$ & $X_{4}$ \\
\hline$p\left(x_{i}\right)$ & 0.2 & 0.1 & 0 & 0.6 \\
$\bar{p}\left(x_{i}\right)$ & 0.2 & 1 & 0.1 & 0.7 \\
\hline
\end{tabular}

Table 1. Example 2: a metagrid with 4 cells

$$
\underline{P}(Y)=\sum_{i=1}^{n}\left(\underline{p}\left(x_{i}\right) \prod_{j=1}^{i-1} \bar{p}\left(\neg x_{j}\right)\right),
$$

a sum that has a linear number of terms with respect to $n$. We can similarly obtain an upper bound $\bar{P}(Y)$, just inverting the bounds over $p\left(x_{i}\right)$ in the equation. The bounds $\left[\underline{P}\left(Y^{c}\right), \bar{P}\left(Y^{c}\right)\right]$ over the event $Y^{c}=$ "none of the cells of the metagrid are occupied" can then be obtained by complementation, as we have $\underline{P}\left(Y^{c}\right)=1-\bar{P}(Y)$ and $\bar{P}\left(Y^{c}\right)=1-\underline{P}(Y)$.

Example 3. Let us consider a metagrid composed of four cells with probability intervals of occupancy given in Table 1. In this case, we have:

$$
\begin{aligned}
\underline{P}(Y)= & \underline{p}\left(x_{1}\right)+\underline{p}\left(x_{2}\right) \bar{p}\left(\neg x_{1}\right) \\
& +\underline{p}\left(x_{3}\right) \bar{p}\left(\neg x_{1}\right) \bar{p}\left(\neg x_{2}\right) \\
& +\underline{p}\left(x_{4}\right) \bar{p}\left(\neg x_{1}\right) \bar{p}\left(\neg x_{2}\right) \bar{p}\left(\neg x_{3}\right) .
\end{aligned}
$$

Using the fact that $\bar{p}\left(\neg x_{i}\right)=1-\underline{p}\left(x_{i}\right)$, we obtain:

$$
\begin{aligned}
& \underline{P}(Y)=0.2+0.1(1-0.2) \\
& +0(1-0.2)(1-0.1) \\
& +0.6(1-0.2)(1-0.1)(1-0)=0.712
\end{aligned}
$$

Similarly,

$$
\begin{aligned}
\bar{P}(Y)= & \bar{p}\left(x_{1}\right)+\bar{p}\left(x_{2}\right) \underline{p}\left(\neg x_{1}\right) \\
& +\bar{p}\left(x_{3}\right) \underline{p}\left(\neg x_{1}\right) \underline{p}\left(\neg x_{2}\right) \\
& +\bar{p}\left(x_{4}\right) \underline{p}\left(\neg x_{1}\right) \underline{p}\left(\neg x_{2}\right) \underline{p}\left(\neg x_{3}\right) .
\end{aligned}
$$

Thus, we have:

$$
\begin{aligned}
& \bar{P}(Y)=0.2+1(1-0.2) \\
& +0.1(1-0.2)(1-1) \\
& +0.7(1-0.2)(1-1)(1-0.1)=1,
\end{aligned}
$$

giving a probability interval of occupancy equal to $[0.712,1]$, what might indicate that the metagrid is not free. Note that the upper bound is equal to 1 which is quite natural because it is totally plausible for the second cell to be occupied. Similarly, the high lower bound is induced by the fact that the fourth cell is probably occupied. 


\begin{tabular}{c|cccc}
\hline & $Y_{1}$ & $Y_{2}$ & $Y_{3}$ & $Y_{4}$ \\
\hline$\underline{P}\left(y_{i}\right)$ & 0.1 & 0.3 & 0.712 & 0 \\
$\bar{P}\left(y_{i}\right)$ & 0.2 & 0.5 & 1 & 1 \\
\hline
\end{tabular}

Table 2. Example 3: a trajectory composed of four metagrids

\subsection{Inferences along a trajectory}

Having made inferences over the $k$ metagrids $Y_{1}, \ldots, Y_{k}$ along a given trajectory, we are now concerned about selecting the path that the robot should take. A first step for this is to assess how far we could go along a given trajectory. Indeed, if one of the first metagrid is almost surely occupied, it is not very useful to know that the last ones are surely free, as we will have encountered an obstacle before them. Hence, what matters is how soon we are likely to encounter an obstacle on a path. With this reasoning in mind, we propose to assess the lower and upper probability bounds over consecutive events $F_{i}=$ "the $i$ th metagrid is the first non-free", or in variable terms,

$$
F_{i}=\wedge_{j=1}^{i-1} \neg y_{j} \wedge y_{i} \quad i=1, k .
$$

The event $F_{i}$ being actually expressible as a Cartesian product of the subspaces $\left\{y_{i}, \neg y_{i}\right\}$, we have in the probabilistic case that

$$
P\left(F_{i}\right)=p\left(y_{i}\right) \prod_{j=1}^{i-1} p\left(\neg y_{j}\right) .
$$

Getting bounds over this probability is then very easy, since if all the terms are independent, it is enough to just minimize each of them. We thus end up with

$$
\underline{P}\left(F_{i}\right)=\underline{p}\left(y_{i}\right) \prod_{j=1}^{i-1} \underline{p}\left(\neg y_{j}\right)
$$

and similarly for $\bar{P}\left(F_{i}\right)$, just switching the bounds. Computing this bound involves at most $k$ products, and is therefore quite efficient again. Note that we compute also the bounds for an additional event $F_{k+1}=\wedge_{j=1}^{k} \neg y_{j}$ which represents the event "all metagrids are free".

Example 4. Let us consider a trajectory composed of 4 metagrids which probabilities are given in Table 2 (in which the third metagrid could be the one of Example 3).

These values could correspond to the following situation: the first two metagrids are likely to be free, then the sensor detects almost surely an obstacle on the third metagrid, and does not see anything on the last metagrid because of occlusions. We have:

$$
F_{1}=y_{1} \times\left\{y_{2}, \neg y_{2}\right\} \times\left\{y_{3}, \neg y_{3}\right\} \times\left\{y_{4}, \neg y_{4}\right\}
$$




\begin{tabular}{c|ccccc}
\hline & $F_{1}$ & $F_{2}$ & $F_{3}$ & $F_{4}$ & $F_{5}$ \\
\hline$\underline{P}\left(F_{i}\right)$ & 0.1 & 0.24 & 0.28 & 0 & 0 \\
$\bar{P}\left(F_{i}\right)$ & 0.2 & 0.45 & 0.63 & 0.18 & 0.18 \\
\hline
\end{tabular}

Table 3. Example 3: bounds computation for the $F_{i}$

$$
\begin{gathered}
F_{2}=\neg y_{1} \times y_{2} \times\left\{y_{3}, \neg y_{3}\right\} \times\left\{y_{4}, \neg y_{4}\right\} \\
F_{3}=\neg y_{1} \times \neg y_{2} \times y_{3} \times\left\{y_{4}, \neg y_{4}\right\} \\
F_{4}=\neg y_{1} \times \neg y_{2} \times \neg y_{3} \times y_{4} \\
F_{5}=\neg y_{1} \times \neg y_{2} \times \neg y_{3} \times \neg y_{4}
\end{gathered}
$$

Using Esq. (5), we can compute easily the probability bounds of the five events. They are given in Table 3

It should also be noted that the events $F_{1}, \ldots, F_{k+1}$ actually form a partition of the space $\prod_{i=1}^{k}\left\{y_{i}, \neg y_{i}\right\}\left(F_{i} \cap F_{j}=\emptyset\right.$ for any $i, j$, and $\left.\cup_{i=1}^{k+1} F_{i}=\prod_{i=1}^{k}\left\{y_{i}, \neg y_{i}\right\}\right)$, meaning that they can be interpreted as probability bounds over atoms of a Boolean algebra. This means in particular that the set of intervals

$$
\left[\underline{P}\left(F_{i}\right), \bar{P}\left(F_{i}\right)\right], i=1, k
$$

can be interpreted as probability intervals 9 , a well known imprecise probabilistic model for which efficient inference tools do exist. We will use this fact in the next section, when deciding which path a mobile robot should follow in order to maximize its utility.

\subsection{Deciding the best trajectory}

We now consider that we have to choose between several paths $\tau$, each moving over a fixed number of $k$ metagrids whose states are uncertain. To choose the best trajectory, we propose to use a utility function quantifying the fact that a trajectory is usable or not. This utility is defined on the basis of the events $F_{i}$.

A first remark is that the states or atoms $F_{i}, i=1, k+1$ of our decision space are naturally ordered in terms of increasing utility, in the sense that $F_{j}$ (the $j$-th metagrid is the first occupied) happening is worse than $F_{j+1}$ happening. This means, among other things, that the utility function associated to those states should be increasing, i.e., that $u\left(F_{i}\right) \leq u\left(F_{i+1}\right)$ in any case.

In the case of precise probabilities (we have a precise value $P_{j}\left(F_{i}\right)$ for each element of a trajectory $\tau_{j}$ ), the value of a trajectory would then be its expected utility $\mathbb{E}\left(\tau_{j}\right)$, i.e.

$$
\mathbb{E}\left(\tau_{j}\right)=\sum_{i=1}^{k+1} P_{j}\left(F_{i}\right) u\left(F_{i}\right)
$$


and

$$
\underline{\mathbb{E}}\left(\tau_{j}\right)=\sum_{i=1}^{k+1}\left(u\left(F_{i}\right)-u\left(F_{i-1}\right)\right) \underline{P}\left(\left\{F_{i}, \ldots, F_{k+1}\right\}\right),
$$

When the $P\left(F_{j}\right)$ 's become imprecise and are only known to belong to some intervals $\left[\underline{P}\left(F_{j}\right), \bar{P}\left(F_{j}\right)\right]$, this expectation becomes interval-valued for each trajectory $\tau_{j}$. Given the fact that our uncertainty model is expressed as intervals on singletons, the upper and lower probabilities are 2-monotone and thus we can compute the lower and upper expected utilities of each trajectory thanks to a Choquet integral (we refer to ${ }^{9}$ for the details of the derivation). Moreover, as the utilities are always ordered in the same way, computing the bounds of the expected utility is simple as we have:

$$
\underline{P}\left(\left\{F_{i}, \ldots, F_{k+1}\right\}\right)=\max \left(\sum_{l=i}^{k+1} \underline{p}\left(F_{l}\right), 1-\sum_{l=1}^{i-1} \bar{p}\left(F_{l}\right)\right),
$$

238

with $u\left(F_{0}\right)=0$. Similarly

$$
\overline{\mathbb{E}}\left(\tau_{j}\right)=\sum_{i=1}^{k+1}\left(u\left(F_{i}\right)-u\left(F_{i-1}\right)\right) \bar{P}\left(\left\{F_{i}, \ldots, F_{k+1}\right\}\right),
$$

239

and

$$
\bar{P}\left(\left\{F_{i}, \ldots, F_{k+1}\right\}\right)=\min \left(\sum_{l=i}^{k+1} \bar{p}\left(F_{l}\right), 1-\sum_{l=1}^{i-1} \underline{p}\left(F_{l}\right)\right) .
$$

Again, due to our assumptions (increasing and ordered utilities along the $F_{i}$, that

Example 5. Let us consider the utilities given in Table 4. We have chosen to fix negative utilities for the two first metagrids, representing a kind of "security distance" of at least two metagrids, a negative expected utility then corresponding to the fact that if this path is chosen, the mobile robot should preferably brake or stop. If we compute the lower and upper bounds for the trajectory of Example 4 . we have

$$
\begin{aligned}
\underline{\mathbb{E}}(\tau) & =(-20-0) \cdot \underline{P}\left(\left\{F_{1}, \ldots, F_{5}\right\}\right)+(-10-(-20)) \cdot \underline{P}\left(\left\{F_{2}, \ldots, F_{5}\right\}\right)+\ldots \\
& +(20-10) \cdot \underline{P}\left(\left\{F_{5}\right\}\right) \\
& =(-20-0) \cdot 1+(-10-(-20)) \cdot 0.8+\ldots+(20-10) \cdot 0=-8.5
\end{aligned}
$$

and we finally find $[-8.5,1]$, which corresponds to the fact that the second metagrid

As each trajectory is characterized by a lower and an upper expectation, there are several possibilities to make a decision about the best trajectory, as we must now 


\begin{tabular}{cccccc}
\hline$u\left(F_{0}\right)$ & $u\left(F_{1}\right)$ & $u\left(F_{2}\right)$ & $u\left(F_{3}\right)$ & $u\left(F_{4}\right)$ & $u\left(F_{5}\right)$ \\
\hline 0 & -20 & -10 & 0 & 10 & 20 \\
\hline
\end{tabular}

Table 4. Example 3 continuation: utility values

compare intervals and no longer point-valued evaluations. Some classical ways $\frac{31}{10}$ to do so are the following:

- Order 1 . We can say that a trajectory $\tau_{j}$ is better than a trajectory $\tau_{k}$ $\left(\tau_{j} \succ \tau_{k}\right)$ if and only if $\underline{\mathbb{E}}\left(\tau_{j}\right) \geq \overline{\mathbb{E}}\left(\tau_{k}\right)$.

- Order 2. We can say that a trajectory $\tau_{j}$ is better than a trajectory $\tau_{k}$ if and only if $\underline{\mathbb{E}}\left(\tau_{j}\right) \geq \underline{\mathbb{E}}\left(\tau_{k}\right)$ and $\overline{\mathbb{E}}\left(\tau_{j}\right) \geq \overline{\mathbb{E}}\left(\tau_{k}\right)$.

- Order 3 . We can say that a trajectory $\tau_{j}$ is better than a trajectory $\tau_{k}$ if and only if $\underline{\mathbb{E}}\left(\tau_{j}\right) \geq \underline{\mathbb{E}}\left(\tau_{k}\right)$.

- Order 4 . We can say that a trajectory $\tau_{j}$ is better than a trajectory $\tau_{k}$ if and only if $\overline{\mathbb{E}}\left(\tau_{j}\right) \geq \overline{\mathbb{E}}\left(\tau_{k}\right)$.

The two first rules provide a partial order on the trajectories allowing for incomparabilities, whereas the two last rules provide a linear order. Rule 3 is a pessimistic rule, it consists in retaining the best trajectory in the worst case, i.e. the trajectory associated to the highest lower expected utility. Rule 4 on the contrary gives the preference to the highest upper expected utility, adopting in this way an optimistic behaviour. When the order is partial (rules 1 and 2), one can determine a set of non-dominated trajectories:

$$
T^{*}=\left\{\tau_{j} \mid \nexists \tau_{k} \text { such that } \tau_{k} \succ \tau_{j}\right\}
$$

If this set is composed of more than one trajectory and that a unique trajectory is mandatory, several strategies can then be applied. It is for example possible to postpone the decision by asking complementary information on the navigable space. It is also possible to use additional criteria in the decision process, like the proximity to a reference trajectory (using in this case what is commonly known as lexicographic orders 11).

Example 6. Let us suppose that we have 5 possible trajectories, whose lower and upper expected utilities are given in Table 5 and represented in Figure 3 . Using Rule 1 , there are three maximal elements in the partial order induced by the intervals $\left(\tau_{2}, \tau_{4}\right.$, and $\left.\tau_{5}\right)$. Two maximal elements are found using Rule $2\left(\tau_{4}\right.$ and $\left.\tau_{5}\right)$. The pessimistic rule 3 leads to the choice of $\tau_{4}$, whereas Rule 4 chooses $\tau_{5}$.

\section{Illustrative application}

As a proof of concept, we experiment our approach using real data, issued from the platform PACPUS ${ }^{17}$ developed in our lab. The objective of PACPUS is to provide 


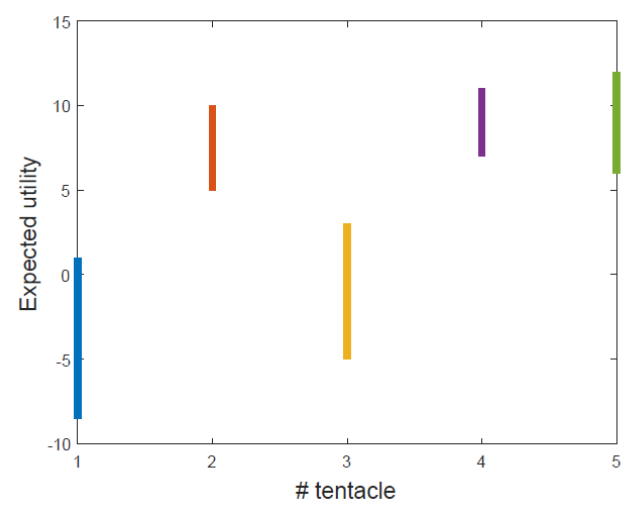

Fig. 3. Representation of the expected utilities of the trajectories

\begin{tabular}{c|ccccc} 
& $\tau_{1}$ & $\tau_{2}$ & $\tau_{3}$ & $\tau_{4}$ & $\tau_{5}$ \\
\hline$\underline{\mathbb{E}}\left(\tau_{j}\right)$ & -8.5 & 5 & -5 & 7 & 6 \\
\hline$\overline{\mathbb{E}}\left(\tau_{j}\right)$ & 1 & 10 & 3 & 11 & 12 \\
\hline
\end{tabular}

Table 5. Bounds of expected utilities of the trajectories

tools and resources for experimenting on intelligent vehicles. We use a Lidar as a perception sensor. This sensor can distinguish between free and occupied space and model it in 2D ( $\mathrm{x}, \mathrm{y}$ coordinates) with respect to the vehicle bodyframe. We used the credal grids generated by a $\mathrm{C}++$ code 19 with data acquired on our experimental vehicle (cf Figure 4). We refer to the provided references for further details about the grid acquisition. We consider a set of 40 different grids (or images). Each grid of $20^{*} 50$ meters is built with uniform cells of size $0.1^{*} 0.1$ meters. Each grid is represented as an image in which one pixel corresponds to one cell, the green color shows the free cells (usable), the red one shows the occupied cells, while the black represents unexplored cells (unknown). The color intensity reflects the certainty degree. For each image (or grid), we generated $n$ possible trajectories, as represented in Figure 5. The shapes of the trajectories are clothoids, that are generated following the approach proposed by Mouhagir et al. 22. The number of generated trajectories depends on internal states of ego-vehicle (speed and yaw rate). This type of trajectory called "tentacle" is indeed well suited to local planning. The final aim is then to automatically select the best trajectory, or "tentacle". In the following we consider $n=5$ trajectories. Generating such a finite set of pre-computed trajectories is quite common when needing real-time decision for objects having a high velocity (rockets, cars, etc.), as it speeds up the decision process. In this case, exploring all solutions 


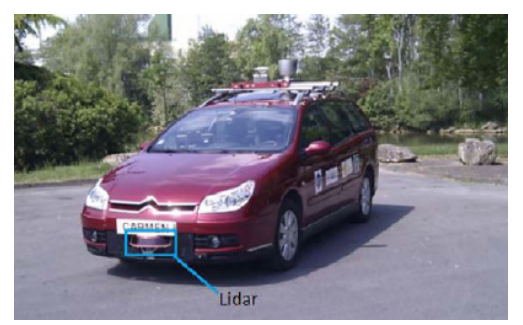

Fig. 4. Experimental vehicle

and trajectories may simply be too costly, even with efficient algorithms at hand.

Each trajectory is composed of several metagrids, each of them are defined as a square encompassing a circle of 3 meters diameter ( $30 * 30$ cells). The security distance is fixed to 15 meters which is equivalent to 5 metagrids along a trajectory. The red circles indicate that the distance of the vehicule to the metagrid is less than the security distance. Each cell X of a metagrid is characterized by a basic belief mass assignment (bba) $m$ on the frame $\mathcal{X}=\{x, \neg x\}$, where $x$ stand for "occupied" and $\neg x$ for "free", that can be easily transformed into lower/upper probabilistic bound\$c This means that for each cell, we retrieve three masses $m(\{x\}), m(\{\neg x\}), m(\mathcal{X})$. Each metagrid, initially of size $30^{*} 30$, was transformed into metagrids of size $6 * 6$ by averaging the masses of adjacent cells.

To have access to a ground truth and to be able to compare methods for choosing the best trajectory, we have used two human "experts" (in this case, experienced drivers from the laboratory) to label the possible trajectories. An example of some evaluations given by one expert is given in Table 6. The instructions given to the experts were to assign a rank to the proposed trajectories, with the convention that 0 means that the trajectory is not at all acceptable (i.e., could lead to an accident), that rank 1 is the best rank, and that the same rank can be given to several trajectories if the expert is not able to distinguish them in terms of drivability. For example, in Table 6, for grid 200, trajectory $\tau_{5}$ is declared unacceptable by the expert, and $\tau_{3}$ is preferred to $\tau_{2}$, which is in turned preferred to $\tau_{1}$ and $\tau_{4}$, without any preference between these two last trajectories. In image 209, $\tau_{4}$ and $\tau_{5}$ are unacceptable, $\tau_{2}$ and $\tau_{3}$ are equally preferred, and $\tau_{1}$ is in the third position.

To apply our method, we convert the evidential grids (which we simply use as an estimation/learning tool) into imprecise probability grids in the following a way: each cell of a metagrid is characterized by an interval of probability $[\underline{p}(x), \bar{p}(x)]$ such that:

$$
\underline{p}(x)=m(\{x\}),
$$

\footnotetext{
${ }^{\mathrm{c}}$ We consider in this paper only normal bbas so that $m(\emptyset)=0$ for any cell of the grid, so we can just treat them as our way to obtain probability bounds, without considering their potential semantic within evidence theory.
} 


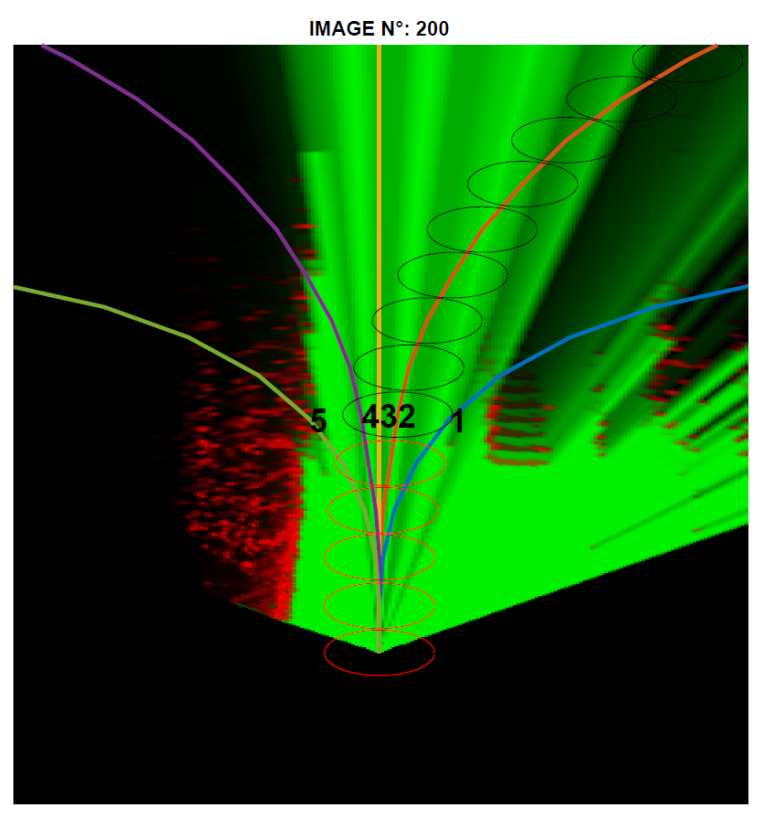

Fig. 5. A credal grid and five possible trajectories

\begin{tabular}{cccccc}
\hline Grid & $\tau_{1}$ & $\tau_{2}$ & $\tau_{3}$ & $\tau_{4}$ & $\tau_{5}$ \\
\hline $\mathbf{2 0 0}$ & 3 & 2 & 1 & 3 & 0 \\
$\mathbf{2 0 9}$ & 2 & 1 & 1 & 0 & 0 \\
\hline
\end{tabular}

Table 6. Example of trajectory evaluation by an expert

and

$$
\bar{p}(x)=m(\{x\})+m(\{x, \neg x\}) .
$$

The decision is based on 12 metagrids per trajectory which corresponds in average to what can be visually evaluated by the expert. As it is partly outside of what can be seen by the Lidar, the first metagrid is not taken into account in the decision process. We have fixed arbitrarily the values of the utility function to -5 for the first four metagrids, and to values linearly spaced between 10 and 70 for the following ones.

As a baseline to compare our method, we also convert the evidential grids into binary grids and use them as follows:

(1) A cell is occupied if the middle of the interval $[\underline{p}(x) ; \bar{p}(x)]$ is greater than 
0.5 .

(2) A metagrid is free if none of the cells that make it up are occupied,

(3) If the rank of the first occupied metagrid is less or equal to 4, then the trajectory is declared unacceptable.

(4) The further away is the first metagrid occupied along a trajectory, the better is the trajectory.

The reason for choosing such a baseline is that it is very simple (hence easily usable by an autonomous car or any mobile robot), but retains very little of our initial, rich information. A key question is therefore to check that retaining such a rich (but computationally more demanding) information does bring some practical advantage. The methods are evaluated according to the two tasks performed during the decision: deciding if a trajectory is acceptable or not and ordering of the remaining trajectories. The methods are thus evaluated according to two different criteria.

For the first task, we use the traditional $F_{\beta}$ measure which is defined by:

$$
F_{\beta}=\frac{\left(1+\beta^{2}\right) \mathrm{TP}}{\left(1+\beta^{2}\right) \mathrm{TP}+\beta^{2} \mathrm{FN}+\mathrm{FP}},
$$

where TP stands for the number of true positives, FN for false negatives and FP for false positives. In the task of recognizing acceptable trajectories, the false negative refer to acceptable trajectories which are wrongly classified as unacceptable, whereas the false positives refer to unacceptable trajectories which are wrongly declared as acceptable. The last error being much more serious and dangerous for a driver than the former, we use a value of $\beta=0.5$ which penalizes more the false positives than the false negatives. Two possible rules have been explored in the experiments:

- Rule 1. trajectory $\tau_{j}$ is classified as acceptable if $\mathbb{E}\left(\tau_{j}\right)>0$;

- Rule 2. trajectory $\tau_{j}$ is classified as acceptable if $\overline{\mathbb{E}}\left(\tau_{j}\right)>0$.

For the second task, we need a criterion to judge the relevance of the trajectory ordering. As explained before, the order relations on the set of trajectories obtained using our method are either total preorders (orders 3 and 4) or partial orders (orders 1 and 2). On the other hand, the evaluations of the experts, expressing their preference about the trajectories with possible indifference, lead to total preorders. A first way to compare the results is to use a general measure to evaluate the discrepancy between preorders, as partial or total orders are special cases of preorders. For this purpose, we use a distance measure between preorders proposed in 15 . Let us use the following notations: $\tau_{j} \succ \tau_{k}$ denotes the fact that $\tau_{j}$ is strictly preferred to $\tau_{k}, \tau_{j} \approx \tau_{k}$ that $\tau_{j}$ and $\tau_{k}$ are indifferent, and $\tau_{j} ? \tau_{k}$ means that $\tau_{j}$ and $\tau_{k}$ are incomparable. The distance between two preorders is defined as the sum of the distances between all pairs of trajectories, using the values indicated in Table 7. These values have been arbitrarly chosen in a set of values respecting axiomatic and common sense rules.

For Orders 3 and 4, and for the binary method, as they lead to total preorders, there is a second way of comparing the results: if indecision is not allowed, each 


\begin{tabular}{ccccc}
\hline & $\tau_{j} \approx \tau_{k}$ & $\tau_{j} \succ \tau_{k}$ & $\tau_{j} ? \tau_{k}$ & $\tau_{k} \succ \tau_{j}$ \\
\hline$\tau_{j} \approx \tau_{k}$ & 0 & 1 & $4 / 3$ & 1 \\
$\tau_{j} \succ \tau_{k}$ & 1 & 0 & $4 / 3$ & $5 / 3$ \\
$\tau_{j} ? \tau_{k}$ & $4 / 3$ & $4 / 3$ & 0 & $4 / 3$ \\
$\tau_{k} \succ \tau_{j}$ & 1 & $5 / 3$ & $4 / 3$ & 0 \\
\hline
\end{tabular}

Table 7. Values of the distance between two preorders

method can be forced to select a best trajectory i.e. a trajectory which is ranked first (there may be multiple ones in case of indifference) in the preorder. We propose in this case to evaluate each method by an accuracy score computed as follows. Let $\hat{\tau}_{i}$ denote the prediction of a method. For each predicted trajectory, the individual accuracy is obtained as:

$$
a c c_{i}=\left\{\begin{array}{l}
\frac{1}{r_{i}} \text { if } \hat{\tau}_{i} \text { is acceptable for the expert } \\
0 \text { else }
\end{array}\right.
$$

where $r_{i}$ denote the rank of $\hat{\tau}_{i}$ in the expert order. The total accuracy Acc is obtained as the mean of the individual accuracies.

It must be noticed that, in contrast with the IP method, the binary method often provides several trajectories equally ranked at the first place because the order depends only on the number of metagrids on the trajectories supposed to be free. In case of a tie, we propose thus two strategies: either to retain the best rank among the set of best trajectories (which gives clearly an advantage to the binary method) or to break the tie by randomly choosing a trajectory in the best trajectories, which seems fairer for the comparison, as in principle we do not have access to the expert "ground truth".

Results are presented in Table 89 , and 10 . In Table 8 is presented the value of $F_{\beta}$ averaged on the 40 images for the two rules and the binary approach. We can see that the best score for $F_{\beta}$ is achieved using Rule 2. This rule consists in eliminating trajectories for which the utility is for sure less than zero. This confirms that using negative utilities for the first metagrids is a useful feature, as it is an efficient filter. Note that results of our method and the binary one are not very different. As future works, we could try to optimize/change utilities so that they provide optimal $F_{\beta}$ on a training set, yet this would significantly increase the method complexity, with an unclear benefit.

Much more differences are found in the second task. Table 9 presents the results obtained for the ordering task (after using Rule 2 for selecting the acceptable trajectories). It gives the average distance between the expert orders and the automatic orders. It can be seen that, for both experts, the order obtained using Order 4 leads to a smallest distance with respect to the expert order and that it outperforms the 
simple binary method. Interestingly, this indicates that once the undesirable (unacceptable) trajectories have been removed from the possible choices, the optimistic behaviour seems to be in very good accordance with the observed expert behaviours. Order 1 seem to be too conservative, as could be reasonably expected, while orders 2 and 3 end up in similar distances. It should however be emphasized that we are systematically comparing all orders to total preorders, meaning that it is hard, from our experimental setting, to assess the utility of the incomparabilities induced by orders 1 and 2, in particular the refine an imprecise selection. Exploring this aspect is the matter of future methodological and experimental work, for example to allow experts to express such incomparabilities.

In Table 10. we compare the results of the task consisting in choosing only one trajectory. We report three results for the simple binary method: the maximum accuracy which corresponds to breaking ties in choosing systematically the trajectory associated to the best result (the one ranked highest by the expert), the minimum accuracy which corresponds to choosing systematically the worst result and, finally, a random guess among the possible best trajectories. Without surprise, the results of the binary method in which the maximum accuracy is retained gives the best results but it may be seen that Order 4 outperforms the random guess and provides results which are close to maximum accuracy. Another thing is that the span between the minimum and maximum accuracies can be quite large, thus indicating that a refined modelling of uncertainty may be helpful ot better discriminate the trajectories.

\begin{tabular}{cccc}
\hline Expert & $F_{\beta}$ (rule 1$)$ & $F_{\beta}$ (rule 2$)$ & $F_{\beta}$ (binary) \\
\hline 1 & 0,8934 & $\mathbf{0 , 9 1 1 8}$ & 0,9034 \\
2 & 0,8768 & $\mathbf{0 , 9 4 6 0}$ & 0,9429 \\
\hline
\end{tabular}

Table 8. Classification unacceptable/acceptable: average $F_{\beta}$ for the three methods

\begin{tabular}{cccccc}
\hline Expert & Order 1 & Order 2 & Order 3 & Order 4 & Binary \\
\hline 1 & 9,7 & 6,31 & 6,85 & $\mathbf{2 , 8 1}$ & 6,84 \\
2 & 9,86 & 6,4 & 6,2 & $\mathbf{3 , 6 1}$ & 7,32 \\
\hline
\end{tabular}

Table 9. Ordering results: mean distance between the expert and the proposed order 


\begin{tabular}{cccccc}
\hline Expert & Order 3 & Order 4 & Binary (min) & Binary (random) & Binary (max) \\
\hline 1 & 0,6995 & 0.9125 & 0,5372 & 0.7855 & 0.9251 \\
2 & 0,6663 & 0,8792 & 0,4422 & 0,6446 & 0,9625 \\
\hline
\end{tabular}

Table 10. Best trajectory results: accuracy of the methods

\section{Conclusion}

In this article, we have introduced the notion of credal occupancy grids, that to the best of our knowledge was never studied before. The advantage of such grids is their flexibility in terms of uncertainty modelling, as well as their consistency with traditional probabilistic models, that they extend. To show how such grids could be used in practice, we have proposed a model to make cautious inferences for robot trajectory planning from uncertain information provided in the form of such credal occupancy grids. The proposed model allows one to accurately represent the available information while controlling the computational complexity by the use of particular events of the decision space. In particular, the proposed method is relatively simple and makes very clear assumptions about the uncertainty modelling and reasoning parts, as well as about the decision process. This has in our opinion two key advantages: it makes the whole method more acceptable and explainable to third parties, and it is easy to revisit, question, or modify the different components of the method.

We have illustrated the behaviour of the proposed approach using simple examples, and have tested it using real data, in the context of autonomous vehicles, comparing it favourably with a simple baseline approach. These first results indeed show that the approach is interesting thanks to a greater flexibility in the decision process. To completely validate the approach, a greater number of experts and a larger number of grids and situations in the experimental part would be needed. Future research could include also the automatic learning of the utility function and the integration of other criteria in the decision process like the similarity to a reference trajectory.

We also plan to augment the expressivity of the method, for example by better differentiating cell occupancy information, distinguishing between completely usable cells, not-fully usable cells (e.g. for vehicles, side-walks, emergency lines) and completely unusable cells (with obstacles). Such distinctions could be important elements to deal with complex situations possibly leading to accidents, but would increase the method complexity. Another important avenue of research is how dependence information could be embedded in the inferences. Indeed, so far we have considered the cells and their uncertainty model to be independent, which is an acceptable simplifying assumption in first approximation, but is not true in general. 
Finally, while we considered here the problem of making real-time inferences on a pre-selected number of trajectories by using DNF Boolean formulas, it would be interesting to investigate other ways to handle either the planning problem or to obtain the normal forms of the formulas, that could prove useful in those cases where computational time should remain acceptable but is not constrained by realtime and by physical constraints induced by velocity and limiting the number of trajectories one could consider (e.g., slow moving robots in risky environments). For instance, one could consider finding an arbitrary optimal sequence of cells as a trajectory from point $A$ to point $B$, taking inspiration from recent works on inferences in HMM 8 or from works extending PODMP 14 to the imprecise framework. Concerning Boolean formulas, we mainly used DNF formulas and mentioned BDDs as another way to obtain compact representations of Boolean functions, but it would interesting to make a general exploration of the interplay between different ways of compiling semantically equivalent Boolean formulas ${ }^{\mathbf{7}}$ and their corresponding computational complexity, notably those compilations that have an efficient imprecise counterpart such as the recent Sentential Decision Diagrams 18 .

\section{Acknowledgements}

This work was carried out in the framework of Equipex ROBOTEX (ANR-10EQPX-44-01) and Labex MS2T (ANR-11-IDEX-0004-02), which were funded by the French Government, through the program "Investments for the future" managed by the National Agency for Research.

1. F. Aguirre, S. Destercke, D. Dubois, M. Sallak, and C. Jacob. Inclusion-exclusion principle for belief functions. International Journal of Approximate Reasoning, 55(8):17081727, 2014.

2. Thomas Augustin, Frank PA Coolen, Gert De Cooman, and Matthias CM Troffaes. Introduction to imprecise probabilities. John Wiley \& Sons, 2014.

3. Endre Boros and Peter L Hammer. Pseudo-boolean optimization. Discrete applied mathematics, 123(1-3):155-225, 2002.

4. R. E. Bryant. Symbolic boolean manipulation with ordered binary-decision diagrams. ACM Computing Surveys (CSUR), 24(3):293-318, 1992.

5. Davide Ciucci. Orthopairs: A simple and widely usedway to model uncertainty. Fundamenta Informaticae, 108(3-4):287-304, 2011.

6. C. Coue, C. Pradalier, C. Laugier, T. Fraichard, and P. Bessiere. Bayesian occupancy filtering for multitarget tracking: an automotive application. The International Journal of Robotics Research, 25(1), pp. 19-30, 2006.

7. Adnan Darwiche and Pierre Marquis. A knowledge compilation map. Journal of Artificial Intelligence Research, 17:229-264, 2002.

8. Jasper De Bock and Gert De Cooman. An efficient algorithm for estimating state sequences in imprecise hidden markov models. Journal of Artificial Intelligence Research, 50:189-233, 2014.

9. L.M. de Campos, J.F. Huete, and S. Moral. Probability intervals: a tool for uncertain reasoning. International Journal of Uncertainty, Fuzziness and Knowledge-Based Systems, 2:167-196, 1994. 
10. S. Destercke and D Dubois. Special cases. In Introduction to Imprecise Probabilities, chapter 4, pages 79-92. John Wiley \& Sons, Ltd, 2014.

11. P.C. Fishburn. Exceptional paper-lexicographic orders, utilities and decision rules: A survey. Management science, 20(11):1442-1471, 1974.

12. J. Fortin, D. Dubois, and H. Fargier. Gradual numbers and their application to fuzzy interval analysis. IEEE Transactions on Fuzzy Systems, 16(2):388-402, 2008.

13. Wolfgang Gatterbauer and Dan Suciu. Oblivious bounds on the probability of boolean functions. ACM Transactions on Database Systems (TODS), 39(1):1-34, 2014.

14. Hideaki Itoh and Kiyohiko Nakamura. Partially observable markov decision processes with imprecise parameters. Artificial Intelligence, 171(8-9):453-490, 2007.

15. K. Jabeur, J.-M. Martel, and S. B. Khélifa. A distance-based collective preorder integrating the relative importance of the group's members. Group Decision and Negotiation, 13(4):327-349, 2004.

16. Paul A Jensen and Mandell Bellmore. An algorithm to determine the reliability of a complex system. IEEE Transactions on reliability, 18(4):169-174, 1969.

17. Heudiasyc Laboratory. Plateforme PACPUS. https://pacpus.hds.utc.fr/ Accessed:2019-11-04

18. Lilith Mattei, Alessandro Antonucci, Denis Deratani Mauá, Alessandro Facchini, and Julissa Villanueva Llerena. Tractable inference in credal sentential decision diagrams. International Journal of Approximate Reasoning, 125:26-48, 2020.

19. J. Moras, C. Cherfaoui, and P. Bonnifait. Moving objects detection by conflict analysis in evidential grids. In IEEE Intelligent Vehicles Symposium (IV), pages 1122-1127, Baden-Baden, Germany, June 2011.

20. J. Moras, V. Cherfaoui, and P. Bonnifait. Credibilist occupancy grids for vehicle perception in dynamic environments. In IEEE International Conference on Robotics and Automation (ICRA), pages 84 - 89, Shangai, China, May 2011.

21. H. Moravec and A. Elfes. High resolution maps from wide angle sonar. In IEEE International Conference on Robotics and Automation (ICRA), volume 2, pages 116 - 121, St Louis, USA, March 1985.

22. Hafida Mouhagir, Reine Talj, Véronique Cherfaoui, François Aioun, and Franck Guillemard. Evidential-Based Approach for Trajectory Planning With Tentacles, for Autonomous Vehicles. IEEE Transactions on Intelligent Transportation Systems, pages $1-12,2019$.

23. S. Noykov and C. Roumenin. Occupancy grids building by sonar and mobile robot. Robotics and Autonomous Systems, 55(2):162-175, 2007.

24. G. Oriolo, G. Uliviy, and M. Vendittelli. Fuzzy maps: A new tool for mobile robot perception and planning. Journal of Robotic Systems, 14(3):179-197, 1997.

25. Guillermo Owen. Multilinear extensions of games. Management Science, 18(5-part2):64-79, 1972 .

26. D. Pagac, E. Nebot, and H. Durrant-Whyte. Evidential approach to map-building for autonomous vehicles. IEEE Transactions on Robotics and Automation, 14(4):623-629, 1998.

27. Chandima Dedduwa Pathiranage, Keigo Watanabe, and Kiyotaka Izumi. T-s fuzzy model adopted slam algorithm with linear programming based data association for mobile robots. Soft Computing, 14(4):345, 2010.

28. A. Saffiotti. The uses of fuzzy logic in autonomous robot navigation. Soft Computing, 1(4):180-197, 1997.

29. P. Smets. Decision making in the TBM: the necessity of the pignistic transformation. International Journal of Approximate Reasoning, 38:133-147, 2005.

30. G. Tanzmeister and D. Wollherr. Evidential grid-based tracking and mapping. IEEE 
December $\quad 4, \quad 2020 \quad 10: 57$ WSPC/INSTRUCTION $\quad$ FILE

Vehicle_IP_revised_ijufks

Transactions on Intelligent Transportation Systems, 18(6):1454-1467, 2017.

31. M.C.M. Troffaes. Decision making under uncertainty using imprecise probabilities. International Journal of Approximate Reasoning, 45:17-29, 2007.

32. P. Walley. Statistical reasoning with imprecise probabilities. Chapman and Hall, New York, 1991

33. T. Yang and V. Aitken. Evidential mapping for mobile robots with range sensors. IEEE Transactions on Instrumentation and Measurement, 55(4):1422-1429, 2006. 